\title{
Expert rowers' motion analysis for synthesis and technique digitalization
}

\author{
Alessandro Filippeschi ${ }^{*} \quad$ Emanuele Ruffaldi $^{*}$ \\ (*) Scuola Superiore Sant'Anna, TECIP, Pisa, Italy \\ E-mail: a.filippeschi@sssup.it,e.ruffaldi@sssup.it
}

\begin{abstract}
Four expert rowers' gestures were gathered on the SPRINT rowing platform with the aid of an optic motion tracking system. Data were analyzed in order to get a digital representation of the features involved in rowing. Moreover, these data provide a dataset for developing digital models for rowing motion synthesis. Rowers were modeled as kinematic chains, data were processed in order to get position and orientation of upper body limbs. This representation was combined with SPRINT data in order to evaluate features found in the literature, to find new ones and to build models for the generation of rowing motion. The analysis shows the effectiveness of the motion reconstruction and two examples of technique features: stroke timing and upper limbs orientation during the finish phase.
\end{abstract}

\section{Introduction}

Rowing require athletes to excel both in fitness and motion accuracy. Coaches and rowers focus their attention mostly on fitness status, embedding technique training in protocols aimed at optimizing fitness. This approach influenced and was influenced by technology: devices for monitoring physiological status were already used by trainers in the 1970s, whereas specific technologies for biomechanical evaluation of rowing gesture are still not commonly exploited. Technique is indeed evaluated mostly qualitatively by inspection of video tapes. Technologies' improvements allowed only recently to get quantitative information about rowers' motion in out-door rowing, these data were exploited to describe experts' gestures and technique features ([4]). Parallel to the development of devices for outdoor rowing technique evaluation, in-door rowing simulators were developed since 1960s. These devices typically reproduce load well enough to be used for rowing training but they don't provide users with some impor-

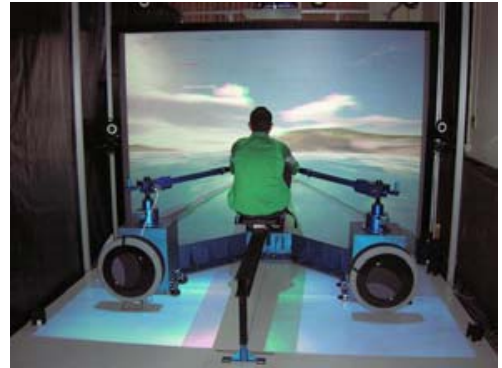

Figure 1: The SPRINT system.

tant kinematic features (e.g the Concept2 ergometer ${ }^{1}$, the world reference for rowers). Only recently rowing simulators providing accurate kinematics features were proposed to the market ${ }^{2}$, whereas the most sophisticated ones have been recently developed by researchers ([7], [8]) and exploit the potential of virtual environments for training. In-door rowing systems can be used to capture and analyze rowers performance ([2],[3]). The SPRINT $^{3}$ system [7] (Fig. 1) was designed to perform in-depth analyses which aim in a first step at developing digital models of technique features, in a second one at using such models for training people. This paper shows an experiment carried out to extract rowing features from experts' performances. In this way a database useful for virtual rowers motion generation and to evaluate stroke timing and arm-forearm orientation in the finish phase (the phase in which arms pull oars, see [1] for rowing stroke detailed nomenclature) was created. Moreover, for the latter feature, a dependency of orientation on pace we did not found in the literature is presented.

\footnotetext{
${ }^{1}$ http://www.concept2.com/

${ }^{2}$ e.g. Biorower: http://www.biorower.com/

${ }^{3}$ http://www.skills-ip.eu/row/
}

This is an Open Access article distributed under the terms of the Creative Commons Attribution-Noncommercial License 3.0, which permits unrestricted use, distribution, and reproduction in any noncommercial medium, provided the original work is properly cited. 


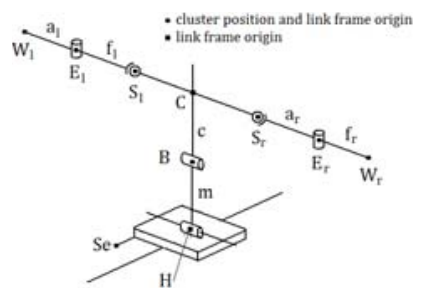

Figure 2: Scheme of the calibration pose.

\section{Method}

Four expert rowers (males, aged $22.2 \pm 4$ ) were asked to row on the SPRINT platform. The task consisted of performing seven technique faults and rowing correctly, it is worth noting that rowers cross their hands when sculling, and they put left one over the right regardless their handedness. Experts had to voluntary perform faults at two severity levels (minor and large) and at three paces: 18, 24 and 30 strokes/minute(spm). They had finally to row correctly at paces between 18 and $36 \mathrm{spm}$ equally spaced. The protocol was therefore composed of seven faults by two levels by three paces, that is 42 blocks, plus seven blocks of correct rowing. Each block recording was manually started by the experimenter when the expert decided he was correctly accomplishing the task, the block ended by the experimenter after he had counted ten strokes, of which at least half correctly performed. Rest time was decided by the expert in order to avoid fatigue to influence mastery of technique. Experts were asked about thier fatigue status after the experiment. In addition to variables available in SPRINT, the VICON motion capture system was used for upper body motion tracking, an in-depth technique analysis indeed require detailed knowledge of back and arms motion [4]. Cluster based motion tracking was selected for its stability and reliability: rowers wore clusters of markers on their wrists, elbows, shoulders and back, one additional cluster was placed on the sliding seat. Before starting the protocol, a calibration of the system on the current expert was performed asking him to wait steady in a previously defined calibration pose. Figure 2 shows a scheme of the calibration pose along with clusters arrangement and nomenclature. All data were available in Matlab Simulink and sampled at $125 \mathrm{~Hz}$. The system was completed by a Virtual Environment (VE) projected in front of the expert where, superimposed to a single sculling scenario, they could see the pace they were rowing at as a number in the middle of the screen.

\section{Processing}

Data processing was composed of three steps: filtering, integration with calibration data and reconstruction of kinematics. Analyzed data were wrists (W), elbows (E), shoulders (S), back (B) and seat (Se) positions with respect to a world frame whose $\mathrm{x}$-axis is aligned with the seat rail and y-axis vertical. Only the upper body limbs were considered in the model: midriff, chest, arms and forearms were modeled as rigid links connected respectively by hinge, ball and hinge joints.

Filtering. Cluster data needed to be filtered because of occurred problems, in particular missing tracking or mix up of clusters. Mistaken data were removed, gaps were filled by means of spline interpolating correct data. Filtered data arrays contained the same number of samples of the original ones, the correct samples being placed in the same position as in the original arrays. Since correctness of strokes were judged by the expert himself while rowing, data were visually inspected to remove partial and mistaken strokes, in this phase were removed only strokes clearly incorrect.

\subsection{Calibration}

Data recorded in the calibration phase were used for correcting positions of elbows and back. Arms and forearms were assumed to be horizontal, back and chest to be vertical. The middle point $\mathrm{C}$ of shoulders displacement was computed and used to establish the offset of middle point $\mathrm{H}$ of hips positions from seat position: $\mathrm{H}$ was considered to be vertically aligned with $H$, hence obtaining horizontal displacement from Se, vertical displacement was measured for each rower. Elbows and back positions were translated to belong respectively to arm-forearm lines $\overrightarrow{S W}$ and $\overrightarrow{H C}$ (an approximation of the spine), needed offsets $\Delta \mathbf{E}_{l}, \Delta \mathbf{E}_{r}$ and $\Delta \mathbf{B}$ were then stored in order to translate elbows and back position for all the samples, Figure 3 shows an example of such correction in a pose different from the calibration one.

\subsection{Reconstruction}

Reconstruction consists of calculating positions and orientations of the links as well as joint angles from recorded data. For this purpose each link was replaced with a frame, shown in Fig. 4. Origins and axes unit vectors (called axes themselves in the following) are hence defined:

- Chest: origin in $\mathbf{C}=\left(\mathbf{S}_{l}+\mathbf{S}_{r}\right) / 2, \mathbf{x}_{c}$ along the shoulder line, $\mathbf{z}_{c}$ vertical, $\mathbf{y}_{c}$ towards anterior direction. 


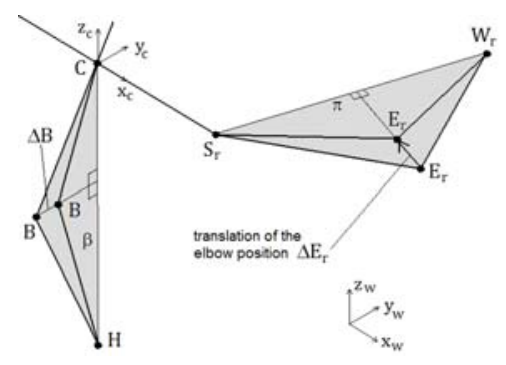

Figure 3: Correction of elbows $\left(\boldsymbol{\Delta} E_{i}\right)$ and back $\left(\boldsymbol{\Delta}_{B}\right)$ clusters positions.

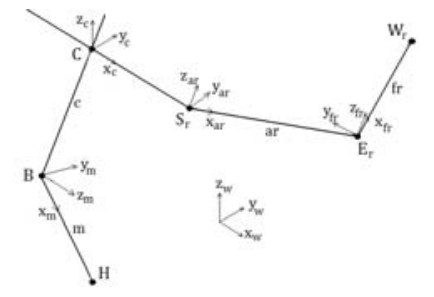

Figure 4: Representation of the link frames (left side not shown).

- Arms: origin in $\mathbf{S}_{i}, \mathbf{x}_{a i}$ along the arm, $\mathbf{y}_{a i}$ in the S-E-W planes ( $\pi_{l}$ and $\pi_{r}$ planes), $\mathbf{z}_{a i}=\mathbf{x}_{a i} \times \mathbf{y}_{a i}$.

- Forearm: origin in $\mathbf{E}+\Delta \mathbf{E}_{i}, \mathbf{x}_{f i}$ along the forearm, $\mathbf{y}_{f i}$ in the S-E-W plane ( $\beta$ plane), $\mathbf{z}_{f i}=\mathbf{x}_{f i} \times \mathbf{y}_{f i}$.

- Midriff: origin in $\mathbf{B}+\Delta \mathbf{B}, \mathbf{x}_{m}$ along $\overrightarrow{B C}, \mathbf{y}_{m}$ in the C-B-H plane, $\mathbf{z}_{m}=\mathbf{x}_{m} \times \mathbf{y}_{m}$.

The reconstruction of body limbs started from a reference configuration (the same of calibration pose with vertical arms instead of horizontal), all body frame axes were assigned subscript 0 (e.g $\mathbf{x}_{f l 0}$ for left forearm $\mathrm{x}$ axis) when referred to this configuration. Each current axis $\mathbf{x}_{i}$ can be obtained from the reference one $\mathbf{x}_{i 0}$ by means of the link rotation matrix $R_{i}$ defined by $\mathbf{x}_{i}=R_{i} \mathbf{x}_{i 0}$. Rotation matrices are therefore obtained as

$$
R_{i}=\left[\begin{array}{lll}
\mathbf{x}_{i} & \mathbf{y}_{i} & \mathbf{z}_{i}
\end{array}\right]\left[\begin{array}{lll}
\mathbf{x}_{i 0} & \mathbf{y}_{i 0} & \mathbf{z}_{i 0}
\end{array}\right]^{T}
$$

Limbs frames and rotation matrices allowed to represent all joints variables, elbows angles are given by $\theta_{e i}=\arccos \left(-\mathbf{x}_{a i}^{T} \mathbf{x}_{f i}\right)$; back limbs angle $\theta_{b}$ is defined similarly, shoulder joint rotation was defined by Euler angles for the sequence flexion $\theta_{s y}$, abduction $\theta_{s x}$ and rotation $\theta_{s z}$, that is, arm frame can be obtained from reference one by rotating respectively around $\mathbf{x}_{a 0}$ axis, obtained $\mathbf{y}_{a}$, obtained $\mathbf{z}_{a}$. Euler angles relation with $R_{a}$ components are

$$
\begin{aligned}
& \theta_{\text {six }}=\operatorname{atan} 2\left(\frac{R_{s i}(3,2)}{\cos \theta_{\text {siy }}}, \frac{R_{\text {si }}(3,3)}{\cos \theta_{\text {siy }}}\right) \\
& \theta_{\text {siy }}=-\arcsin R(3,1) \\
& \theta_{\text {siz }}=\operatorname{atan} 2\left(\frac{R_{\text {si }}(2,1)}{\cos \theta_{\text {siy }}}, \frac{R_{\text {si }}(1,1)}{\cos \theta_{\text {siy }}}\right)
\end{aligned}
$$

which never degenerates with rowing gesture data.

\section{Technique features}

In this section the map of two important ([5],[6]) technique features on the data are shown. The first is cylce phases timing the other one, related to the finish phase, is the $\pi$ planes orientation with respect to gravity. They have been sought in order to get a quantitative index of skilled performance.

\subsection{Timing}

Timing of back swing and arms bending onset are an index of the rhythm rowers are keeping and of their ability in using their muscles in the correct sequence. Strokes were segmented at the beginning of the drive phase, when legs start pushing, oar horizontal angle $\phi$ was used for detecting the beginning of the stroke: the passage of $\dot{\phi}$ from negative to positive gave the stroke onset reference time. Back swing onset time $T_{b}$ estimation was based on $\beta$ plane orientation with respect to gravity, in particular a threshold on $\dot{\beta}$ sufficed to detect back swing. Arms bending was detected by comparing current $\overrightarrow{B C}$ length $u l$ with the calibration one $u l_{0}$, a threshold on $u l / u l_{0}$ allowed to discriminate arms motion onset time $T_{a}$. Drive phase elapsed time $T_{d}$ was finally marked by the passage of $\dot{\phi}$ from positive to negative. Given this framework, timing indexes were calculated as

$$
t_{b}=T_{b} / T_{d} \quad \text { and } \quad t_{a}=T_{a} / T_{d}
$$

in order to clear linear dependency of $T_{i}$ indexes on pace from the analysis. Timing indexes dependence on pace was sought mixing all expert data. Figure 6 shows results of analysis. Consistency of stroke structure was checked on drive and recovery phases times, Fig. 5 shows typical profiles of drive and recovery phases elapsed time ([6]) with the only difference that drive-recovery times ratio is smaller. Fig. 6 shows a Scatter plot of timing data along with regression curves, linearity for both was checked obtaining $R^{2}=0.29$ for back timing and $R^{2}=0.01$ for arms timing. 


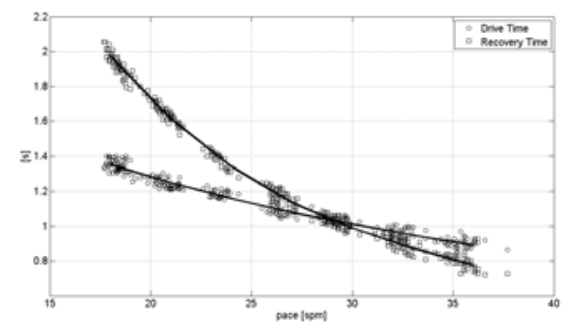

Figure 5: Drive and recovery times against pace.

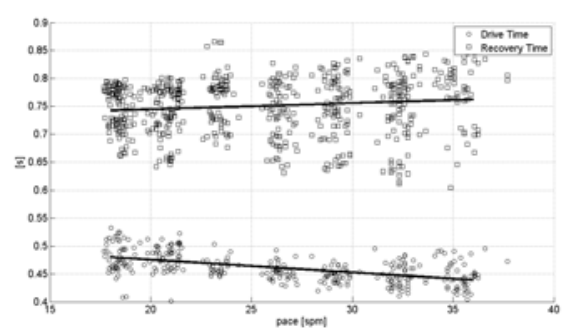

Figure 6: Plot of timing against pace for the four rowers.

\subsection{Finish arm-forearms orientation}

Finish phase effectiveness depends on rowers ability to keep pressure on the blade. If $\pi_{i}$ plane is too vertical blades are likely to exit the water too early (washing out fault). Only the samples between $T_{a}$ and $T_{d}$ were considered to belong to the finish phase. Given $n_{i}$ the unit vector perpendicular to $\pi_{i}$, for each sample and $v=\left[\begin{array}{lll}0 & 0 & 1\end{array}\right]$ the vertical unit vector we define the finish angle $\theta_{f i}=\arccos \left(\mathbf{v}^{T} \mathbf{n}_{i}\right)$ which gives the orientation of $\pi_{i}$ with respect to gravity. Figure 7 shows angles $\theta_{f l}$ for the four experts. Despite very different body sizes angles are similar for rowers 1-3, rowers 4 declared to row with elbows a little too low (that is $\theta_{f l}$ too high), it was confirmed by his coach. Data of rowers 1-3 were then tested for a dependency of $\theta_{f i}$ angles on pace. Intra-subject t-test showed the rise of $\theta_{f l}$ with pace to be statistically relevant: $\mathrm{p}$-values $(\alpha=0.01)$ for 18-27, 18-36 and 27-36 comparisons are over 0.70 except for 27-36 comparison of rower 2 .

\section{Conclusions and future work}

The present work allowed to create a database of expert rower motion. Such a database is being used to make a digital representations of technique features, to be used as a motion source for virtual rowers in the virtual environment SPRINT is provided with, and finally to synthesize rowing motion in order to develop a rowing gesture generator flexible on rowers size and training demands. This paper has also shown two examples
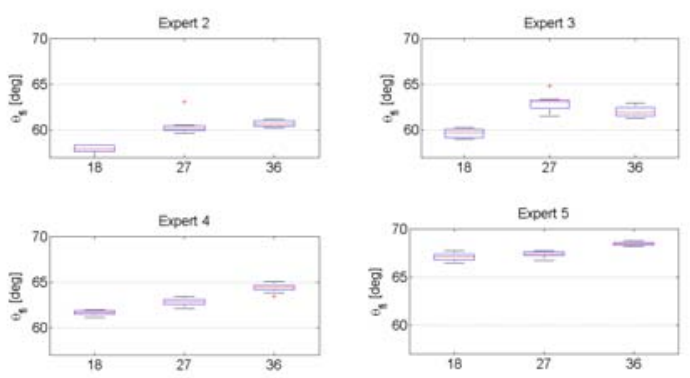

Figure 7: Finish angles $\theta_{f i}$ for the four rowers.

of how the database can be used for mapping known technique features on the training platform and how a numerical representation of gesture may lead to the discovery of unknown expert gesture features.

Acknowledgments This work was supported by the SKILLS Integrated Project (IST-FP6 \#035005, http://www.skills-ip.eu) funded by the European Commission.

\section{References}

[1] FISA rowing handbook. FISA, 2006.

[2] A. Baudouin and D. Hawkins. Investigation of biomechanical factors affecting rowing performance. Journal of Biomechanics, 37(7):969-976, 2004.

[3] S. Fothergill, R. Harle, and S. Holden. Modeling the Model Athlete: Automatic Coaching of Rowing Technique. In Proc. of the Joint IAPR, pages 372381. Springer, 2008.

[4] V. Kleshnev. Rowing biomechanics. available online http://www.biorow.com/, 2006.

[5] V. Kleshnev. Boat acceleration, temporal structure of the stroke cycle, and effectiveness in rowing. Proc. of the Institution of Mech. Eng., Part P: Journal of Sports Engineering and Technology, 224:6374, 2010.

[6] V. Nolte. Rowing Faster. Human Kinetics, 2005.

[7] E. Ruffaldi, A. Filippeschi, A. Frisoli, C. A. Avizzano, B. Bardy, D. Gopher, and M. Bergamasco. Sprint: a training system for skills transfer in rowing. In T. Gutirrez and E. Snchez, editors, SKILLS09, Bilbao,Spain, December 2009. CEIT.

[8] J. von Zitzewitz, P. Wolf, V. Novakovic, M. Wellner, G. Rauter, A. Brunschweiler, and R. Riener. Real-time rowing simulator with multimodal feedback. Sports Technology, 1(6), 2008. 